\title{
Total positive curvature of circular DNA
}

\author{
Bohr, Jakob; Olsen, Kasper Wibeck
}

Published in:

Physical Review E

Link to article, DOI:

10.1103/PhysRevE.88.052714

Publication date:

2013

Document Version

Publisher's PDF, also known as Version of record

Link back to DTU Orbit

Citation (APA):

Bohr, J., \& Olsen, K. W. (2013). Total positive curvature of circular DNA. Physical Review E, 88(5), 052714. https://doi.org/10.1103/PhysRevE.88.052714

\section{General rights}

Copyright and moral rights for the publications made accessible in the public portal are retained by the authors and/or other copyright owners and it is a condition of accessing publications that users recognise and abide by the legal requirements associated with these rights.

- Users may download and print one copy of any publication from the public portal for the purpose of private study or research.

- You may not further distribute the material or use it for any profit-making activity or commercial gain

- You may freely distribute the URL identifying the publication in the public portal

If you believe that this document breaches copyright please contact us providing details, and we will remove access to the work immediately and investigate your claim. 


\title{
Total positive curvature of circular DNA
}

\author{
Jakob Bohr* and Kasper W. Olsen ${ }^{\dagger}$ \\ DTU Nanotech, Building 345Ø, Ørsteds Plads, Technical University of Denmark, 2800 Kongens Lyngby, Denmark
}

(Received 15 January 2013; revised manuscript received 15 August 2013; published 20 November 2013)

\begin{abstract}
The properties of double-stranded DNA and other chiral molecules depend on the local geometry, i.e., on curvature and torsion, yet the paths of closed chain molecules are globally restricted by topology. When both of these characteristics are to be incorporated in the description of circular chain molecules, e.g., plasmids, it is shown to have implications for the total positive curvature integral. For small circular micro-DNAs it follows as a consequence of Fenchel's inequality that there must exist a minimum length for the circular plasmids to be double stranded. It also follows that all circular micro-DNAs longer than the minimum length must be concave, a result that is consistent with typical atomic force microscopy images of plasmids. Predictions for the total positive curvature of circular micro-DNAs are given as a function of length, and comparisons with circular DNAs from the literature are presented.
\end{abstract}

DOI: 10.1103/PhysRevE.88.052714

PACS number(s): 87.14.gk, 87.15.A-, 02.40.Hw

\section{INTRODUCTION}

An early triumph in understanding the topology of DNA is rooted in White's theorem, also associated with Călugăreanu and Fuller [1-4]. The theorem describes the relationship between linking, twist, and writhe, i.e., $\mathrm{Lk}=\mathrm{Tw}+\mathrm{Wr}$. Interestingly, White's theorem does not say anything about the material properties of DNA, nor about its preference for having a right-handed helical structure (B-DNA). This preference breaks the mirror symmetry and makes DNA chiral, which gives rise to special properties, e.g., a coupling between strain and twist. Intuitively, the double-stranded helical geometry of DNA suggests the existence of fascinating structural phenomena. One recent example in a study of supercoiled DNA is long-ranged dynamical effects between two distant plectonemes [5]. Theoretical aspects of the physics of polymers and DNA have been reviewed in detail $[6,7]$. Here, some topological restrictions for closed curves will be studied. The motivation is to better understand what determines the shape of closed loops of chain molecules.

\section{TWIST NEUTRALITY}

An example of two linked space curves $\alpha$ and $\beta$ is shown in Fig. 1. For two space curves that are closed and oriented, the Gauss linking number $\operatorname{Lk}(\alpha, \beta)$ is a topologically conserved integer [8]. The linking number describes the number of times the two curves interlink each other. The self-linking number $\operatorname{Lk}(\alpha, \vec{N})$ of a regular space curve $\alpha$ requires a differentiable field of unit normal vectors $\vec{N}$. We assume the curve to be smooth to the order of at least $\mathcal{C}^{3}$. Together, the unit tangent, the unit normal, and the unit binormal vectors frame the curve. White's theorem applies equally well to framed curves and the total twist becomes the twist integral of the vector field $\vec{N}$ with a factor of $2 \pi$. Another number which describes the vector field $\vec{N}$ is the winding number. The winding number requires to be measured relative to something, i.e., we must have two vector fields defined at the same time. Here we will use the

\footnotetext{
*jabo@nanotech.dtu.dk

${ }^{\dagger}$ kasol@ nanotech.dtu.dk
}

Frenet frame $\vec{N}_{F}$, actually a modified Frenet frame $\vec{N}_{m F}$ [9], as a reference.

Let $\theta$ be the angle between the two local frames, i.e., $\theta=$ $\arccos \left(\vec{N} \cdot \vec{N}_{m F}\right)$, and let $s$ be a natural parameter for the curve $\alpha$ then the winding number $\Phi$ of $\vec{N}$ relative to the modified Frenet frame becomes

$$
\Phi=\frac{1}{2 \pi} \int_{\alpha} \frac{\partial \theta}{\partial s} d s .
$$

One could have chosen another reference frame. For example, for the writhe frame, with this type of reference frame the winding number of the normal vector field becomes equal to its linking number [10]. With the modified Frenet frame as a reference, the winding number becomes

$$
\Phi=\operatorname{Lk}(\alpha, \vec{N})-\operatorname{Lk}\left(\alpha, \vec{N}_{m F}\right) .
$$

Therefore $\Phi$ is a topologically conserved integer. Let us now consider a bundle of frames $\vec{N}(u), u \in \mathbf{R}$, which are differentiable in the vicinity of $u=0$ and for which $\vec{N}(0)=\vec{N}$. It follows from differentiation of Eq. (1) that

$$
\int_{\alpha} \frac{\partial^{2} \theta(s, u)}{\partial u \partial s} d s=0 .
$$

This is a remarkable simple result that we denote the twist neutrality lemma. In other words, the differential changes in the rate of the winding progression must obey a zero sum rule.

In the following we will make the assumption that the straintwist coupling for a short stretch of DNA only depends on its strain, curvature, and torsion $(\sigma, \kappa, \tau)$. Hence, at small strains the incremental change in the rate of winding $\delta$ will depend only on $\kappa$ and $\tau$,

$$
\delta(\kappa, \tau)=\left.\frac{\partial^{2} \theta}{\partial \sigma \partial s}\right|_{\sigma=0} .
$$

The physics which underlines this assumption is the relatively local nature of the interactions within DNA between its constituent atoms. After all, it is their interactions that determine the molecular structure as well as its reaction to strain. In Eq. (4) we have chosen not to make the orientation of the double helix, i.e., $\theta$, enter the equation. This means that material properties are considered uniform over about a pitch 


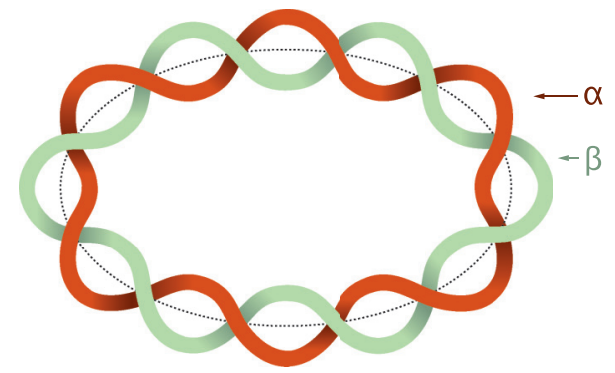

FIG. 1. (Color online) An example of two linked space curves $\alpha$ and $\beta$ is sketched as a representation of a microcircular plasmid.

of the double helix which is between 10 and 11 base pairs (bp) long. For circular DNA one obtains

$$
\int_{\mathrm{DNA}} \delta(\kappa, \tau) d s=0
$$

a restriction that constrains and reduces the number of possible closed space curves (conformations) that double-stranded DNA can make. They are not only topologically restricted by White's theorem, but they are also geometrically restricted because of Eqs. (4) and (5). Thus, strained nonconforming paths would force the DNA to depart from Eq. (5). In the worst case, this could break one, or both, strands of the DNA molecule, in milder cases it could lead to local detachments of the two strands from each other, i.e., local melting of the DNA, while yet milder cases would be approaching insignificant violations of Eq. (5). Denaturation bubbles have been observed in atomic force microscopy (AFM) images of circular DNA $[11,12]$; their closure dynamics has been studied by dynamics simulations [13].

\section{PLANAR PLASMIDS}

What are the implications for closed planar curves, such as plasmids deposited on a substrate? Long plasmids can display many crossings such as supercoils and plectonemic structures [14]. For shorter plasmids the phenomenon of cooperative kinking at distant sites has been studied [15]. The simplest cases are planar plasmids with no self-crossings, i.e., the centerline of the DNA is topologically equivalent to a circle. Recall that straight DNA winds when stretched, i.e., it rotates opposite of the unwinding direction (overwinding) [16,17]. In the present context it means that $\delta>0$ for $\kappa=0$. A straight section of DNA can therefore not satisfy the above discussed twist neutrality criterion. Can a circle of DNA satisfy it? We assume that $\delta$ decreases monotonically as a function of the curvature $1 / R_{0}$ [18]. It means that plasmids that are truly circular, and not just called circular because they are loops, come in one specific size with the plasmid length $L_{0}=2 \pi R_{0}$. Consider a plasmid of length $L$ different from $L_{0}$. We series expand $\delta$ to include the linear turn, i.e.,

$$
\delta=\lambda\left(|\kappa|-\kappa_{0}\right),
$$

where $\kappa$ is the curvature with sign as conventionally used for two-dimensional curves, $\kappa_{0}=1 / R_{0}$ is the curvature of the circle of radius $R_{0}$, and $\lambda$ is a constant that determines the coupling between twist and curvature. Since the curve is assumed planar, $\delta$ is independent of the torsion $\tau$.
The twist neutrality criterion then becomes

$$
\int_{0}^{L}\left(|\kappa(s)|-\kappa_{0}\right) d s=0,
$$

where $\kappa(s)$ is the curvature measured along the plasmid, and $s$ the corresponding arc length. Therefore, we obtain

$$
\int_{0}^{L}|\kappa| d s=2 \pi \frac{L}{L_{0}} .
$$

The integral in Eq. (8) over a closed curve is always greater than or equal to $2 \pi$ : This is Fenchel's inequality [19]. Consequently, there are no possible solutions for plasmids with a length shorter than $L_{0}$. Hence, shorter plasmids are required to be linear, or to melt and become circular single strands.

\section{SHAPES}

What are the possible shapes of plasmids which satisfy the "first-order" twist neutrality criterion Eq. (8) with a length $L$ larger than the smallest plasmid length $L_{0}$ ? The shape must be concave as the integral of the total curvature equals $2 \pi$ exactly when the plasmid is convex [20]. To find a possible shape we cannot use a circle or an ellipse as they are both convex. One concave curve, chosen here because of its relatively simple mathematical expression, is the Cassini oval, which is given by the polar equation

$$
r^{4}-2 a^{2} r^{2} \cos (2 \theta)=b^{4}-a^{4},
$$

where $a$ is half the distance between the two foci, and $b$ the square root of the product of the distances to the two foci. The shape of the Cassini ovals depends on the ratio $\epsilon=a / b$. For $\epsilon<1$ the shape is a single loop with an oval (convex) or peanut (concave) shape, and for $\epsilon=1$ the lemniscate is produced. Figure 2(a) shows a peanut-shaped Cassini curve which is a solution to Eq. (8) with $L=1.5 L_{0}$. The parts of the Cassini oval where the curvature is negative (the progression of the curve is clockwise) are clearly visible. However, the Cassini ovals are not good descriptors of circular DNA which generally are more random in appearance. DNA has a propensity for more randomness, which was demonstrated in an AFM study of the bond correlation function [21]. In this AFM study it was shown that the halfway bond correlation was not fully antiparallel, a result which infers some amount of randomness. By a simple random procedure choosing 15 points and generating a closed curve with a spline, a somewhat more realistic (though not perfect) concave curve is drawn [see Fig. 2(b)]. For this curve $L=1.46 L_{0}$. Figure 2(c) show the trace of an example of a circular plasmid [21]. Further, the concave nature of closed plasmids is also consistent with (a)

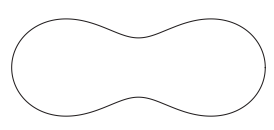

(b)

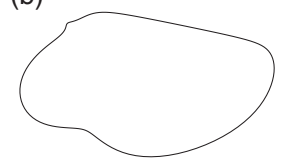

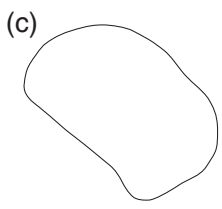

FIG. 2. Examples of concave shapes. (a) A Cassini oval with $L / L_{0}=1.50$, (b) a more random curve generated with the help of 15 seed points and a spline, $L / L_{0}=1.46$, and (c) a path of a minicircle of DNA with 676 bp traced from Ref. [21]. 
the existence of the type of kinks observed in circular DNA $[22,23]$.

\section{TOTAL POSITIVE CURVATURE}

For the general case of a concave curve which satisfies Eq. (8) one has

$$
I_{-}=\int_{0}^{L} \kappa_{-} d s=\frac{L-L_{0}}{L_{0}} \pi .
$$

The integral is the total negative curvature without sign. Here $\kappa_{-}$is equal to $|\kappa|$ when $\kappa<0$, otherwise $\kappa_{-}$is zero. This shows that the total negative curvature must be a linear function of the length $L$. Likewise, using a corresponding definition for $\kappa_{+}$, one can calculate a total positive curvature,

$$
I_{+}=\int_{0}^{L} \kappa_{+} d s=2 \pi+\frac{L-L_{0}}{L_{0}} \pi,
$$

hence $I_{+}-I_{-}=2 \pi$ and $I_{+}+I_{-}=2 \pi L / L_{0}$.

These predictions [Eqs. (10) and (11)] can be experimentally tested by plotting $I_{+}$vs $L$, e.g., in a scatter plot. For the generation of the plot, the total positive curvature $I_{+}$and total negative curvature $I_{-}$was obtained for various circular plasmids (see Table I). The numbers are obtained by digitizing AFM images. The digitized path describes a large polygon and the two total curvatures are subsequently found as the sum of the signed outer angles in the polygon. The advantages of this approach are that slight errors in the digitization will cancel each other on curved stretches of the plasmids. Nevertheless, the numbers are subject to uncertainty, for example, from the limited resolution of the AFM images. Figure 3 shows the results obtained from these digitizations of AFM images of plasmids (pOK14 [24], pSP73 [24], pUC19 [12], pBR322 [25], pEGFP-C1 [26], and two minicircles [21]). The obtained numbers are listed in Table I. The shaded area of the scatter plot indicates a value of $L_{0}$ between 171 and $621 \mathrm{bp}$. Presumably, some of the scatter will disappear when

TABLE I. Positive and negative curvature for circular plasmids. First column: Plasmid name. Second column: Plasmid length (number of base pairs). Third column: Reference for publication. Fourth column: $I_{+}$. Fifth column: $I_{-}$.

\begin{tabular}{lcccc}
\hline \hline Plasmid & {$[\mathrm{bp}]$} & Ref. & $I_{+}$ & $I_{-}$ \\
\hline Minicircle 1 & 241 & {$[21]$} & 7.31 & 1.02 \\
Minicircle 1 & 241 & {$[21]$} & 7.57 & 1.28 \\
Minicircle 1 & 241 & {$[21]$} & 7.57 & 1.29 \\
Minicircle 2 & 676 & {$[21]$} & 13.22 & 6.95 \\
Minicircle 2 & 676 & {$[21]$} & 12.66 & 6.38 \\
pOK14 & 2134 & {$[24]$} & 20.77 & 14.48 \\
pSP73 & 2462 & {$[24]$} & 20.58 & 14.30 \\
pUC19 & 2686 & {$[12]$} & 25.38 & 19.10 \\
pUC19 & 2686 & {$[12]$} & 34.44 & 28.16 \\
pBR322 & 4361 & {$[25]$} & 35.03 & 28.74 \\
pEGFP-C1 & 4700 & {$[26]$} & 26.91 & 20.62 \\
pEGFP-C1 & 4700 & {$[26]$} & 29.46 & 23.18 \\
pEGFP-C1 & 4700 & {$[26]$} & 32.55 & 26.27 \\
pEGFP-C1 & 4700 & {$[26]$} & 32.84 & 26.55 \\
pEGFP-C1 & 4700 & {$[26]$} & 49.39 & 34.10 \\
\hline \hline
\end{tabular}

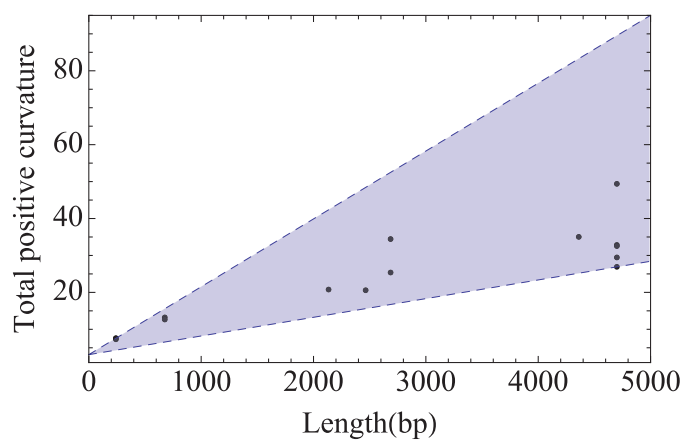

FIG. 3. (Color online) Scatter plot of obtained total positive curvature for plasmids of various lengths from 241 to 4700 base pairs. The shaded area formed by the outer points corresponds to a value of $L_{0}$ between 171 and 621 base pairs.

a more refined method for obtaining the experimental value of the total signed curvatures is developed.

\section{DISCUSSION AND CONCLUSION}

Tubular models offer a simple way to obtain an estimate for $L_{0}$. In a study of their geometry we recently found that a tubular model of B-DNA has no strain-twist coupling when the diameter of the centerline $2 R$ is $156 \AA$ [27]. The corresponding estimate of $L_{0}$ is about $124 \mathrm{bp}$. A tubular model may not capture all numerical aspects of the molecular structure of DNA and this adds to the uncertainty of the estimate. The range for the lower bound $L_{0}$ as obtained from the scatter plot is in reasonable agreement with lengths recently found for small loops of double-stranded DNA in a study of extrachromosomal micro-DNAs [28]. They identified tens of thousands of short circular DNA in mouse tissue as well mouse and human cell lines with an overall size distribution in the range 80-2000 bp with a strong propensity for lengths peaked at around 150 and 300 bp for the human DNA and at 180, 360, 540, 720, and 900 bp (plus perhaps two more peaks) for mouse DNA. Circular single-stranded loops were reported as being yet shorter. The apparent tendency for an integer multiple of a preferred length can, in the perspective of the above analysis, be suggestive of micro-DNAs that coil multiple times when in vivo or are forming plectonemic conformations.

Theoretically, the shape of elastic ring polymers has been considered [29,30]. At biological temperatures DNA is fairly close to the melting temperature of the double strand; the melting temperature depends in detail on such properties as the salt concentration of the aqueous solution and on the sequence of the DNA. Therefore, thermal fluctuations in DNA are omnipresent. Fluctuations in DNA miniplasmids have been modeled as a Kirchhoff-Clebsch rod with no strain [31]. It would be interesting to study excitations and fluctuations, e.g., breathing modes, and to include strain and stretching in detailed studies of the different kinds of modes and their stability in closed loops of chiral filaments [32-34]. It would also be interesting to include higherorder curvature terms in the twist neutrality condition. This includes second-order terms known from elastica and bending energies [35]. 


\section{ACKNOWLEDGMENTS}

The present work was initiated at the Isaac Newton Institute during the workshop "Topological Aspects of DNA Function and Protein Folding." We would like to thank Andrea
Cavalli, Giovanni Dietler, Aleksandre Japaridze, Fridolin Okkels, and Andrzej Stasiak for discussions and Ralf Metzler for correspondence. This work is supported by the Villum Foundation.
[1] J. H. White, Am. J. Math. 91, 693 (1969).

[2] G. Călugăreanu, Czech. Math. J. 11, 588 (1961) (see http://dml.cz/handle/10338.dmlcz/100486?show=full).

[3] F. B. Fuller, Proc. Natl. Acad. Sci. U.S.A. 68, 815 (1971).

[4] W. F. Pohl, Indiana Univ. Math. J. 17, 975 (1968).

[5] M. T. J. van Loenhout, M. V. de Grunt, and C. Dekker, Science 338, 94 (2012).

[6] M. D. Frank-Kamenetskii and A. V. Vologodskiǐ, Sov. Phys. Usp. 24, 679 (1981).

[7] C. Micheletti, D. Marenduzzo, and E. Orlandini, Phys. Rep. 504, 1 (2011).

[8] R. L. Ricca and B. Nipoti, J. Knot Theory Ramifications 20, 1325 (2011).

[9] The modified Frenet frame $\vec{N}_{m F}$ : The Frenet frame requires curvature to be nonzero everywhere. This unnecessarily restricts the class of curves which can be considered. Instead we will consider a modified Frenet frame where the curvature is allowed to be zero at discrete points. The framing is then differentially patched together from sections of Frenet frames, and sections where the normal vector points in the opposite direction of $\vec{N}_{F}$, whichever provides a differential framing. See the discussion of the ribbon frame in J. Bohr and S. Markvorsen, PLoS One 8, e74932 (2013)

[10] M. R. Dennis and J. H. Hannay, Proc. R. Soc. London, Ser. A 461, 3245 (2005).

[11] J.-H. Jeon, J. Adamcik, G. Dietler, and R. Metzler, Phys. Rev. Lett. 105, 208101 (2010).

[12] J. Adamcik, J.-H. Jeon, K. J. Karczewski, R. Metzler, and G. Dietler, Soft Matter 8, 8651 (2012).

[13] A. K. Dasanna, N. Destainville, J. Palmeri, and M. Manghi, Europhys. Lett. 98, 38002 (2012).

[14] N.-K. Lee, T. Schmatko, P. Muller, M. Maaloum, and A. Johner, Phys. Rev. E 85, 051804 (2012).

[15] T. A. Lionberger, D. Demurtas, G. Witz, J. Dorier, T. Lillian, E. Meyhöfer, and A. Stasiak, Nucl. Acids Res. 39, 9820 (2011).
[16] T. Lionnet, S. Joubaud, R. Lavery, D. Bensimon, and V. Croquette, Phys. Rev. Lett. 96, 178102 (2006).

[17] J. Gore, Z. Bryant, M. Nöllmann, M. U. Le, N. R. Cozzarelli, and C. Bustamante, Nature (London) 442, 836 (2006).

[18] K. Olsen and J. Bohr, New J. Phys. 14, 023063 (2012)

[19] W. Fenchel, Math. Ann. 101, 238 (1929).

[20] J. W. Milnor, Ann. Math. 52, 248 (1950).

[21] G. Witz, K. Rechendorff, J. Adamcik, and G. Dietler, Phys. Rev. Lett 101, 148103 (2008).

[22] W. Han, M. Dlakic, Y. J. Zhu, S. M. Lindsay, and R. E. Harrington, Proc. Natl. Acad. Sci. U.S.A. 94, 10565 (1997).

[23] Z. Wei, Z. Haijun, and O.-Y. Zhong-Can, Phys. Rev. E 58, 8040 (1998).

[24] D. Li, Z. Yang, B. Lv, and T. Li, Bioorg. Med. Chem. Lett. 22, 833 (2012).

[25] G. Witz and A. Stasiak, Nucleic Acids Res. 38, 2119 (2009).

[26] V. Bagnacani, V. Franceschi, M. Bassi, M. Lomazzi, G. Donofrio, F. Sansone, A. Casnati, and R. Ungaro, Nat. Commun. 4, 1721 (2013).

[27] J. Bohr and K. Olsen, Phys. Rev. Lett. 108, 098101 (2012).

[28] Y. Shibata, P. Kumar, R. Layer, S. Willcox, J. R. Gagan, J. D. Griffith, and A. Dutta, Science 336, 82 (2012).

[29] K. Alim and E. Frey, Phys. Rev. Lett. 99, 198102 (2007).

[30] K. Ostermeir, K. Alim, and E. Frey, Phys. Rev. E 81, 061802 (2010).

[31] I. Tobias, Biophys. J. 74, 2545 (1998).

[32] K. M. Dempsey and I. Gladwell, Appl. Numer. Math. 20, 211 (1996).

[33] A. E. Zakrzhevskii, V. F. Tkachenko, and V. S. Khoroshilov, Int. Appl. Mech. 46, 1420 (2011).

[34] M. Upmanyu, H. L. Wang, H. Y. Liang, and R. Mahajan, J. R. Soc. Interface 5, 303 (2008).

[35] J. Langer and D. A. Singer, J. Diff. Geom. 20, 1 (1984), http://projecteuclid.org/euclid.jdg/1214438990. 